\title{
Quando os Discentes se Expressam: Um Relato de Experiência da Criação de um Podcast para Aumentar o Engajamento e Troca de Conhecimento em Tempos de Pandemia
}

\author{
Carlos Silva $^{1}$, Kennedy Nunes ${ }^{1}$, Nathasha Pinto ${ }^{1}$, Maikon Costa ${ }^{1}$, \\ Alana Araujo ${ }^{1}$, Simara Rocha ${ }^{1}$, Geraldo Braz Junior ${ }^{2}$ e Luis Rivero ${ }^{1,2}$ \\ ${ }^{1}$ Programa de Educação Tutorial do Curso de Ciência da Computação (PETComp) \\ Universidade Federal do Maranhão (DEINF/UFMA) - São Luís, MA - Brasil \\ ${ }^{2}$ Programa de Pós-Graduação em Ciência da Computação (PPGCC), Universidade \\ Federal do Maranhão (UFMA) - São Luís, MA - Brasil. \\ \{cvs.silva, kennedy.anderson, nathasha.pinto, maikon.keslley, \\ alana.cca\}@discente.ufma.br, \{simara.rocha, geraldo.braz, \\ luis.rivero\}@ufma.br
}

\begin{abstract}
Improving student engagement and facilitating access to information has become more relevant in the educational context. Due to the social isolation generated by the COVID-19 pandemic, educational resources such as podcasts and videos can be exploited to achieve this goal. In order to keep students updated and engaged during remote teaching, the PETComp group at the Federal University of Maranhão has assembled a team to develop a podcast on content related to Information Technology. This paper presents the production process of the podcast episodes. After preparing the material and launching the podcast on digital platforms, students' opinions about it were collected through a questionnaire. The results indicate that the podcast contributed to making content created by the students available in a relaxed way, allowing for updates on relevant topics and the engagement of listeners.
\end{abstract}

Resumo. Melhorar o engajamento de discentes e facilitar o acesso à informação têm se tornado mais relevantes no contexto educacional. Em virtude do isolamento social gerado pela pandemia da COVID-19, recursos didáticos como podcasts e vídeos podem ser explorados para alcançar este objetivo. Com o intuito manter os discentes atualizados e engajados durante o ensino remoto, o grupo PETComp da Universidade Federal do Maranhão montou uma equipe para desenvolver um podcast sobre conteúdos relacionados a Tecnologia da Informação(TI). Este artigo apresenta o processo de produção dos episódios do podcast. Após a preparação do material e lançamento do podcast em plataformas digitais, foi coletada a opinião dos discentes sobre o mesmo através de um questionário. Os resultados indicam que o podcast contribuiu para disponibilizar conteúdos construídos pelos discentes de forma descontraída, permitindo a atualização em temas relevantes e o engajamento dos ouvintes.

\section{Introdução}

A exposição de ideias, conhecimento e notícias deixou de ser uma atividade exclusivamente presencial e passou a alcançar patamares globais com o avanço de tecnologias de informação e comunicação, principalmente com a evolução da internet 
[Barros et al. 2020]. Devido ao isolamento social provocado pela pandemia da COVID19, surgiu a necessidade de ampliar o acesso a informações e expandir o uso de mídias no contexto educacional [Vlachopoulos 2020]. Nesse contexto, plataformas de streaming têm sido utilizadas para acesso de forma mais abrangente às diversas categorias de produtos como: músicas, filmes, jogos, séries e jornais [Mota and Coutinho 2016]. Diante do exposto, o Podcast surge como uma derivação das Web Rádios onde há o suporte da Internet que permite a presença de elementos que não ficam apenas no campo sonoro, mas também em diversas formas de interação [Ferreira et al. 2016].

O número de ouvintes de Podcast aumentou em 60 milhões em 2020, de acordo com um relatório divulgado pela plataforma de streaming Spotify ${ }^{1}$. Além disso, segundo Mota e Coutinho (2009), a interação entre os ouvintes do podcast e o criador pode colaborar para a eficácia do entretenimento e da aprendizagem. Existem relatos do uso de podcasts para permitir que os alunos ouçam material relacionado a um curso enquanto fazem outras atividades [Bell et al. 2007]. Para isso, Farias (2020) indica que é necessário planejar as temáticas de cada episódio de acordo com o público alvo, considerando alternativas de interação entre os apresentadores para evitar que o podcast se torne monótono.

Considerando o impacto que um podcast pode ter no consumo de conteúdos educacionais e informativos, o grupo do Programa de Educação Tutorial de Ciência da Computação (PETComp) da Universidade Federal do Maranhão (UFMA) buscou aplicar esta tecnologia para disponibilizar informações relevantes para a formação dos discentes do curso de Ciência da Computação da UFMA, além de incentivar a integração dos mesmos em decorrência do isolamento social provocado pela pandemia da COVID-19. O Programa de Educação Tutorial (PET) é um programa de apoio e melhoria aos cursos de graduação das Instituições de Ensino Superior do país. Ele procura melhorar a qualidade do ensino, visando a integração de atividades extracurriculares que favoreçam a base curricular dos cursos vinculados a iniciativa. Portanto, atividades que permitam desenvolver os pilares de ensino, pesquisa e extensão nos discentes devem ser executadas.

Neste artigo, é apresentado o relato de experiência da criação desse podcast pelos discentes do PETComp do programa PET. O conjunto de discentes que contribuíram para a criação do podcast definiram um processo para proposição e criação de conteúdo, visando fornecer informações sobre o mercado de trabalho, vivência acadêmica, dicas de produtividade e notícias/curiosidades. Após a produção e disponibilização dos episódios, o podcast foi avaliado pela comunidade acadêmica a partir de um questionário. Os resultados apresentados neste trabalho podem auxiliar outros grupos de discentes interessados em preparar conteúdos educacionais com podcast, incorporando as sugestões e evitando os problemas reportados pelos discentes durante a avaliação deste podcast.

O restante deste trabalho está organizado como segue: A Seção 2 apresenta os trabalhos relacionados, indicando como tem sido abordada a realização de podcasts no ensino de Computação. A Seção 3 apresenta o planejamento adotado na realização do podcast do PETComp - UFMA. Já a Seção 4 apresenta os resultados, desafios e discussões encontrados no decorrer da atividade a partir de reuniões de discussão entre a equipe e a partir um questionário aplicado com o público alvo do podcast. Finalmente a Seção 5 apresenta as principais lições aprendidas e conclui deste relato de experiência.

\footnotetext{
${ }^{1}$ Disponível em: https://investors.spotify.com/financials/press-release-details/2020/SpotifyTechnology-SA-Announces-Financial-Results-for-First-Quarter-2020/default.aspx
} 


\section{Trabalhos Relacionados}

A busca por praticidade alinhada à informação vem crescendo a cada dia, como mostram trabalhos sobre o impacto de podcasts nos seus consumidores [Chan-Olmsted and Wang 2020]. Dentre os tipos de mídia procurados, a busca por podcasts aumentou consideravelmente, uma vez que é possível conciliar a atividade de escutar com várias outras [Mota and Coutinho 2016]. Considerando a alta popularidade dos podcasts, vários trabalhos vêm sendo realizados com o intuito de alinhar podcasts com objetivos educacionais. A seguir são apresentados alguns destes trabalhos relacionados.

Em seu trabalho, Ferreira et al. (2016) desenvolveram um podcast como complemento para a disciplina de algoritmos em um curso de graduação em computação. $\mathrm{O}$ podcast, denominado Algoritmo Podcast, discutiu tópicos relacionados com o conteúdo programático da disciplina de algoritmos, como: conceitos, tipos de dados e operadores, variáveis, estruturas, vetores, matrizes e procedimentos e funções. Ao avaliar o podcast do ponto de vista da turma, foi identificada a recepção positiva do podcast, indicando um aumento na motivação ao estudar para a disciplina atavés deste conteúdo.

Por sua vez, o grupo PET do curso de Ciência da Computação da Universidade do Estado do Rio Grande do Norte descreveu sua experiência ao construir um podcast denominado DIcast [Soares et al. 2020]. Este podcast consiste em entrevistas e discussões sobre temas voltados a tecnologia e áreas correlatas. O seu objetivo é promover conteúdo informativo e atual para a comunidade universitária no formato de áudio. Os autores do trabalho explicam o processo de criação do podcast utilizando uma abordagem autônoma.

Outro podcast em que conteúdos de informática foram apresentados é o Radin, desenvolvido pelo Instituto Federal do Goiás como resposta à suspensão das aulas presenciais devido à pandemia da COVID-19 [Geraldes 2020]. No trabalho, os autores descrevem o processo de produção do primeiro episódio do podcast, descrevendo materiais necessários e ferramentas que podem ser utilizadas para a criação de um podcast.

Apesar do interesse da comunidade acadêmica na utilização de podcasts, os autores deste trabalho identificaram poucos relatos do processo de criação dos podcasts desenvolvidos. Além disso, dos relatos identificados, um foi desenvolvido no âmbito de uma disciplina, o que impede sua utilização em um contexto mais abrangente dentro de um curso de computação. Além disso, os relatos de podcasts com o objetivo de disseminar conteúdos genéricos e permitir a integração dos discentes do curso em tempos de isolamento social não apresentam os detalhes do processo de criação e/ou sua avaliação, dificultando aproveitar lições aprendidas para futuras replicações da construção de podcasts para este fim. Neste trabalho, além de apresentar o processo de criação do podcast, são apresentados os resultados da sua avaliação, a partir do ponto de vista dos discentes ouvintes. Na seção a seguir, é apresentada a metodologia aplicada.

\section{Metodologia}

\subsection{Planejamento Geral}

O podcast PodComp surgiu de uma ideia de um integrante do grupo PETComp, através de uma reunião semanal de integrantes com o professor tutor do grupo. A ideia inicial dos discentes era ter um espaço para poder apresentar conteúdos de interesse para a comunidade acadêmica dentro da universidade. Além disso, essa ideia poderia ter um impacto 
positivo considerando que as atividades de ensino iriam ser executadas de forma remota devido à pandemia da COVID-19. Com essa ideia em mente, uma sub-equipe foi criada para formalizar o projeto, fazendo um planejamento do material que seria produzido.

Num período de quatro semanas, ao redor de 4 reuniões (uma por semana) foram realizadas pela equipe designada composta de quatro discentes em que artigos, livros, blogs, periódicos e jornais foram analisados. A análise ocorreu de forma não controlada, discutindo ideias e anotando as mesmas para validar com o professor tutor. O processo foi livre e o objetivo deste momento de planejamento era a sintetização de informações sobre como estruturar um podcast. Primeiramente a equipe buscou responder as seguintes perguntas: "por quê?", "como?" e "para quem?" criar um podcast. Considerando que Gimenes (2015) afirma que estudantes universitários possuem dificuldades de comunicação, pois o ensino é dado de forma conteudista, decidiu-se então encontrar formas de mudar esse cenário por meio do podcast.

Durante esse período, para que o projeto fosse avaliado e aprovado, também foi feito o levantamento de aspectos da organização de um podcast, onde foram analisados quais os requisitos para a criação dessa mídia. Assim, foi realizada uma análise de características de alguns podcasts do Brasil que os discentes consideravam que eram de interesse e poderiam também servir de base para a proposição de um novo podcast na área de computação. Os podcasts analisados foram: P1 = Ciência da Computação, P2 = Hipsters Ponto Tech, P3 = PrimoCast, P4 = Minuto da Produtividade, P5 = Sinapse e P6 = NerdCast. Os discentes ouviram vários episódios dos podcasts selecionados e indicaram elementos que melhoravam a percepção sobre o conteúdo apresentado. Na tabela 1, são apresentadas as características que o PETComp identificou como necessárias.

Tabela 1. Comparativo das características de podcasts

\begin{tabular}{|c|c|c|c|c|c|c|c|c|}
\hline & Requisitos & $\mathrm{P} 1$ & $\mathrm{P} 2$ & P3 & $\mathrm{P} 4$ & P5 & P6 & Total \\
\hline \multirow{6}{*}{$\begin{array}{l}\text { Sonorização, } \\
\text { Efeitos sonoros }\end{array}$} & Música de Entrada & $\mathrm{X}$ & $\mathrm{X}$ & $\mathrm{X}$ & $\mathrm{X}$ & $\mathrm{X}$ & $\mathrm{X}$ & 6 \\
\hline & Música de Encerramento & $\mathrm{X}$ & $\mathrm{X}$ & $\mathrm{X}$ & $\mathrm{X}$ & $\mathrm{X}$ & $\mathrm{X}$ & 5 \\
\hline & Vinheta & $\mathrm{X}$ & $\mathrm{X}$ & $\mathrm{X}$ & $\mathrm{X}$ & $\mathrm{X}$ & $\mathrm{X}$ & 5 \\
\hline & Música de Fundo & & $\mathrm{X}$ & $\mathrm{X}$ & $\mathrm{X}$ & & $\mathrm{X}$ & 4 \\
\hline & Trilhas de transições entre blocos & & $\mathrm{X}$ & $\mathrm{X}$ & & $\mathrm{X}$ & $\mathrm{X}$ & 4 \\
\hline & Efeitos sonoros específicos (Risada, susto, vaia etc.) & & & $\mathrm{X}$ & & $\mathrm{X}$ & & 2 \\
\hline \multirow{2}{*}{ Mídias Externas } & Criação de mídias em redes sociais, blogs ou sites & $\mathrm{X}$ & $\mathrm{X}$ & $\mathrm{X}$ & $\mathrm{X}$ & & & 4 \\
\hline & Criação de mídias no Youtube & & $\mathrm{X}$ & $\mathrm{X}$ & $\mathrm{X}$ & $\mathrm{X}$ & $\mathrm{X}$ & 5 \\
\hline \multirow{3}{*}{ Referências } & Cita pesquisas científicas e livros & $\mathrm{X}$ & & $\mathrm{X}$ & $\mathrm{X}$ & $\mathrm{X}$ & $\mathrm{X}$ & 4 \\
\hline & Cita Blogs, vídeos, jornais & $\mathrm{X}$ & $\mathrm{X}$ & $\mathrm{X}$ & & $\mathrm{X}$ & $\mathrm{X}$ & 5 \\
\hline & Cita Dados Estatísticos & $\mathrm{X}$ & & $\mathrm{X}$ & & $\mathrm{X}$ & & 3 \\
\hline \multirow{4}{*}{ Formato de Episódio } & SoloCast & & & & $\mathrm{X}$ & & & 1 \\
\hline & Mesa redonda & $\mathrm{X}$ & $\mathrm{X}$ & $\mathrm{X}$ & & $\mathrm{X}$ & $\mathrm{X}$ & 5 \\
\hline & Entrevista & $\mathrm{X}$ & $\mathrm{X}$ & & & & & 2 \\
\hline & StoryTelling & & & & & & & 0 \\
\hline \multirow{2}{*}{ Duração de episódio } & Episódios com duração maior que 30 min & $\mathrm{X}$ & $\mathrm{X}$ & $\mathrm{X}$ & & $\mathrm{X}$ & $\mathrm{X}$ & 5 \\
\hline & Episódios com duração menor que $30 \mathrm{~min}$ & & & & $\mathrm{X}$ & & & 1 \\
\hline \multirow{6}{*}{ Formato de Interação } & Apresentação & & $\mathrm{X}$ & $\mathrm{X}$ & $\mathrm{X}$ & $\mathrm{X}$ & $\mathrm{X}$ & 5 \\
\hline & Alivio Cômico & & & $\mathrm{X}$ & & $\mathrm{X}$ & $\mathrm{X}$ & 3 \\
\hline & Mudanças de pautas e assuntos & $\mathrm{X}$ & $\mathrm{X}$ & $\mathrm{X}$ & & $\mathrm{X}$ & $\mathrm{X}$ & 5 \\
\hline & Revisão do Episódio & & & & & & & 0 \\
\hline & Chamada final para Redes Sociais e outros episódios & $\mathrm{X}$ & $\mathrm{X}$ & $\mathrm{X}$ & $\mathrm{X}$ & $\mathrm{X}$ & $\mathrm{X}$ & 6 \\
\hline & RESULTADO & 12 & 14 & 17 & 9 & 15 & 13 & \\
\hline
\end{tabular}

Após identificar o que seria necessário produzir para que o podcast pudesse ser implementado, a equipe pensou em características que eles gostariam que o podcast ti- 
vesse, do ponto de vista dos discentes do curso em que eles estavam inseridos. Após discussões entre os membros da equipe e o tutor, os discentes indicaram que a linguagem utilizada no podcast deveria ser leve, descontraída e de fácil absorção, visto que a atividade de ouvir este tipo de mídia assumiu um papel significativo no controle de fatores que levam ao estresse e a ansiedade [Phillips 2017]. Além disso, a equipe pensou em apresentar e discutir conteúdos relacionados a Tecnologia da Informação (TI) e relevante para a formação dos discentes, como: mercado de trabalho, vivência acadêmica, dicas de produtividade e notícias/curiosidades. Os discentes também pensaram em alternativas para apresentar tais conteúdos de forma dinâmica para atrair diversos públicos, além da comunidade acadêmica do curso de Ciência da Computação da UFMA.

Considerando o escopo identificado nas reuniões e se baseado em estruturas de podcasts similares, a equipe decidiu dividir o conteúdo do podcast em categorias de episódios, sendo essas: (1) Dicas de Produtividade, que visa apresentar dicas para a otimização das atividades pessoais, estudantis ou no trabalho. (2) Cultura Pop, que visa fazer análises e comentários dos principais filmes/séries, músicas e curiosidades do universo tecnológico ao público interessado; (3) Guia de Sobrevivência na UFMA, que visa apresentar orientações para discentes inexperientes que não conhecem as principais áreas da Universidade, dicas de convivência e curiosidades; e (4) Notícias e Conceitos sobre Tecnologia da Informação(TI), que visa discutir conteúdos sobre a área de tecnologia. Além disso, foi pensado no modo com que se daria a interatividade dos apresentadores e/ou convidados durante os diferentes tipos de episódio. Desta forma, foram pesquisados os principais formatos utilizados em podcasts populares, tendo estas como principais: (1) SoloCast - Formato individual; (2) Entrevista - Sistema pergunta-resposta; (3) Mesa redonda - Uma conversa entre várias pessoas, considerando um sistema de perguntaresposta opcional; e (4) Storytelling - Cobre uma narrativa com uma linha do tempo, real ou fictícia. O grupo decidiu que para os objetivos tratados, os formatos adotados seriam SoloCast, Entrevista e Mesa redonda, e que estes seriam utilizados por conveniência, dependendo da disponibilidade dos membros da equipe e público externo convidado.

Embora um podcast seja gravado normalmente em um ambiente local de trabalho, a equipe optou pela gravação do podcast de forma remota, utilizando a aplicação de voz sobre IP, Discord. Quanto à utilização da aplicação Discord, ela foi escolhida devido à alta frequência de uso em atividades de reuniões de sub-equipes dentro do PETComp, o que evitou dificuldades de aprendizado ou uso da ferramenta por parte da equipe. Finalmente, quanto à periodicidade de lançamento de episódios, foi definida uma frequência semanal ou quinzenal. Dependendo do tempo que os integrantes possuíssem para fazer suas atividades. Este tempo foi pensado viável, pois os discentes conseguiram criar um piloto de um episódio com duração de 10 minutos no prazo de uma semana.

\subsection{Planejamento de Episódios}

Após a definição dos pontos gerais do formato e características do podcast, foi feita a pesquisa do processo de produção dos episódios do mesmo. Nesse contexto, iniciou-se pela definição dos materiais necessários para gravação dos episódios. A busca foi feita em blogs e no Youtube, por discutirem as vantagens e desvantagens dos equipamentos utilizados por profissionais que trabalham com podcasts. Uma vez que cada apresentador faria a gravação na sua casa, foi definido que o microfone de celular seria a melhor opção de equipamento, já que cada um dos apresentadores teria o seu próprio, além de que o 
custeio de um microfone de qualidade não seria viável no momento.

Para que os episódios do PodComp seguissem uma linha coerente do assunto tratado, foi criado um roteiro. Nesse contexto, dois ou três integrantes da equipe, geralmente os apresentadores do episódio em questão, se reuniram para discutir os principais tópicos que seriam tratados em cada bloco de episódio. O roteiro foi dividido da seguinte forma: (1) Apresentação do PodComp e dos apresentadores; (2) Introdução ao assunto; (3) Blocos de discussão dos diferentes subtemas do tema; e (4) Encerramento de episódio, onde o ouvinte também é convidado a acessar as outras mídias do grupo PETComp. Os subtemas do bloco 3 foram decididos a partir da pesquisa do tema principal do episódio em questão. Por exemplo, no caso de um episódio sobre Soft Skills (habilidades não técnicas requeridas no mercado de trabalho), é possível discutir quais tipos existem, o seu impacto e como é possível desenvolve-los ou colocá-los em prática. Os resultados da pesquisa realizada pelos discentes em artigos científicos, artigos de jornais ou blogs definem que conteúdos podem ser discutidos. Vale ressaltar que o conteúdo é validado pelo professor tutor durante as discussões do roteiro. Além disso, as fontes utilizadas como base são citadas na descrição do episódio ou durante as discussões, para que o ouvinte saiba quais fontes serviram de inspiração para o episódio em questão.

A etapa de gravação de um episódio é feita de maneira remota. Nessa etapa, geralmente participam os apresentadores e um integrante responsável por apontar falhas não notadas por quem está falando. O gravador usado para o registro de voz é o do celular de cada participante, onde cada faixa de áudio é guardada para que sejam juntadas na etapa de edição de áudio. O primeiro mês da aplicação do projeto foi voltado para testes, gravações de episódios pilotos e ambientação, já que nenhum dos apresentadores tinha experiências relacionadas a área de comunicação. Ao final da gravação de cada episódio, a mídia era colocada em análise pelo tutor através de uma videochamada e outros integrantes do grupo externos ao projeto, através de feedbacks anônimos. Alguns dos exemplos de comentários feitos nestas reuniões de feedback pelo tutor e pelos discentes do grupo PETComp externos ao podcast foram: Feedback 1: "Gostei muito de como vocês falaram e relataram as coisas. Acho que está faltando ser um pouco mais natural, mas como é o primeiro episódio acho difícil acontecer na primeira tentativa. Achei muito divertido."; Feedback 2: "Gostei das vozes e do jeito como o conteúdo foi exposto, mas falta um pouco de naturalidade na fala e nas interações entre os interlocutores". A partir deles, foi possível abrir espaço para melhorias no modo como se daria a interação dos apresentadores e a forma como os locutores se posicionariam durante as falas.

A edição do áudio foi feita logo após a gravação de cada episódio. Para isso foi necessário pesquisar músicas sem copyright para a música de entrada do programa, vinhetas e efeitos sonoros que seriam usados no episódio. A apresentação do episódio é o primeiro ponto da edição, ela contém a música de entrada, apresentação dos locutores e a chamada da vinheta. Além disso, na exposição do conteúdo são colocadas músicas de fundo e feitas transições das músicas de acordo com o designado no roteiro, isso foi feito seguindo características identificadas em outros podcasts, em que o efeito transmite a ideia de transição no assunto que está sendo exposto. No final do episódio, são usados alguns efeitos sonoros que podem dar a ideia de finalização como: "Aaah que pena" e "Hora da Revisão". Depois dos cortes de áudio, são feitos os tratamentos de ruídos por 
meio do software Reaper ${ }^{2}$. Com isso é apresentada a primeira versão para a revisão, caso seja sugerida alguma alteração ou encontrados erros, o episódio volta para a etapa de edição até estar conforme o planejado, assim gerando novas versões.

Como meio de divulgação da iniciativa do podcast, foi decidido que seriam utilizadas as redes sociais do PETComp UFMA, com aproximadamente 1000 seguidores alunos e ex-alunos do curso de Ciência da Computação da UFMA. As mídias de promoção dos episódios foram criadas usando a plataforma Canva $^{3}$ de criação de templates, devido à facilidade de compartilhamento. O planejamento de lançamento do podcast foi caracterizado por uma grande carga de interação e conteúdo nas plataformas (Instagram e Twitter), onde o público é bastante engajado, focando no aumento de alcance das publicações.

O fim do ciclo da criação de um episódio de podcast é a sua publicação para o público, assim como disponibilizar os materiais feitos a partir dele. Tendo isso em vista, o grupo buscou alternativas para realizar essa publicação. Foi visto que os dois principais modos de distribuição, de acordo com a plataforma Inovação Sebrae Minas ${ }^{4}$, são: (1) Distribuição através de um agregador, ou seja, um programa ou plataforma que faz a hospedagem do áudio do episódio, gerando um Feed RSS (Ferramenta baseada na linguagem $X M L$ e propicia a distribuição de áudios através de um link); e (2) Criação de um Feed RSS em um servidor web, para o qual é preciso ter conhecimentos de $X M L e$ $H T M L$, já que é possível ser linkado a um site. O PETComp decidiu escolher a primeira opção, pois ser a solução com menor custo e maior beneficio, evitando o trabalho de desenvolvimento de um sistema para poder disponibilizar os episódios desenvolvidos. A plataforma usada para a hospedagem do episódio é a versão gratuita da plataforma Cast Box. Nessa versão, ela permite enviar áudios através do Estúdio de Criação, onde ela concede controle no Media Manager, local onde se pode gerenciar podcasts ou episódios, além de acesso às ferramentas de estatísticas e agendamentos de lançamento.

\section{Resultados e Discussão}

Após o planejamento e preparação dos episódios, as mídias relacionadas ao podcast foram disponibilizadas no Instagram do PETComp UFMA 5 . Já os episódios foram disponibilizados ao público na plataforma Spotify ${ }^{6}$. Esta plataforma permite disponibilizar todos os episódios referenciados, além de obter uma análise do público ouvinte. Até o momento da escrita deste artigo, 5 episódios já foram liberados no período de 5 semanas e, de acordo com a análise disponibilizada pela plataforma, houveram 217 inicializações, ou seja, quando o usuário ouve 0 ou mais segundos dos episódios e 118 streamings, ou seja, quando o usuário ouve 60 segundos ou mais dos episódios. Entre eles, há episódios sobre conceituação e aplicação de soft skills, discussão de filmes sobre computação, e sugestões para melhorar a produtividade em tempos de pandemia.

Para entender o impacto do podcast do ponto de vista dos discentes do curso, foi elaborado um questionário. O questionário continha questões fechadas, para indicar o grau de satisfação com atributos do podcast em uma escala likert de 5 pontos (Péssimo,

\footnotetext{
${ }^{2}$ Disponível em: https://www.reaper.fm/

${ }^{3}$ Disponível em: https://canva.com

${ }^{4}$ Disponível em: https://inovacaosebraeminas.com.br/quais-sao-e-como-escolher-um-agregador-depodcast/

${ }^{5}$ Disponível em: https://www.instagram.com/petcompufma/

${ }^{6}$ Disponível em: https://open.spotify.com/show/6fTiqItsIWohIacVtUv0Dn
} 
Ruim, Regular, Bom, ótimo) e questões discursivas sobre sugestões ou críticas em relação ao podcast. Ao todo, 13 discentes ( 12 homens e 1 mulher) com idades entre 19 e 23 anos responderam ao questionário. Destes, 12 indicaram que ouviam podcasts em plataformas como Spotify, Google, Youtube e App Jovem Nerd, com tempo médio semanal de 30 a 50 minutos. Os discentes indicaram que souberam do podcast a partir de recomendações de amigos e redes sociais. A seguir, são apresentados os resultados obtidos ao analisar as respostas destes discentes.

A Tabela 2 apresenta os resultados das questões fechadas feitas no questionário. Segundo os discentes, a qualidade do áudio, efeitos sonoros, a interação entre os apresentadores e a relevância dos temas abordados para a formação dos discentes foi considerada boa ou ótima. Vale ressaltar que alguns discentes acharam a qualidade do áudio e a interação dos apresentadores regular durante suas avaliações. Para entender o que poderia ser melhorado nesse contexto, foram analisadas as questões abertas do questionário.

Com relação ao áudio, os discentes indicaram que interferências como ruídos ou barulho dificultavam o entendimento das falas. Além disso, alguns discentes reclamaram sobre o áudio baixo dos apresentadores e/ou edição, pois algumas falas eram colocadas muito próximas umas das outras. Por sua vez, no que diz respeito à interação dos apresentadores, alguns discentes indicaram que alguns trechos dos episódios eram muito formais e que era necessário ser mais descontraído ou simular uma conversa para não parecer roteirizado. Outros discentes indicaram que em alguns momentos, o nervosismo dos participantes podia gerar gaguejamentos. Com relação à qualidade dos efeitos sonoros, os discentes informaram que estavam adequados, mas poderia haver mais efeitos para destaque de alguns pontos, como palmas. Outros, por sua vez, indicaram que há necessidade de definir um volume adequado para os efeitos para não destoar do restante do áudio e falas. Finalmente, com relação aos temas abordados, embora os discentes não tenham apontado nenhum problema, a maioria dos discentes indicou temas que gostariam que fossem discutidos nos próximos episódios dos podcast. Entre os temas citados, podem ser listados: computação aplicada, mercado de trabalho, a universidade e o engajamento, entrevistas com professores, diversidade na computação, pesquisas na área de computação, dicas de plataformas e ferramentas de ensino, diferença entre a academia e indústria de software e segurança e lei geral de proteção de dados. Alguns dos trechos que indicam a opinião dos discentes sobre os pontos levantados são apresentados a seguir.

"As vezes as trocas de falas parecem muito bruscas não parecendo tanto com uma conversa." - Participante P03 sobre Qualidade do Áudio.

”Acredito que como estamos em um período remoto essa interação pode ser prejudicada, os hosts poderiam criar cenários de forma virtual que permitam a descontração, sinto que falta essa leveza na fala dos hosts." - Participante P06 sobre a Interação dos Apresentadores.

"... poderia ter alguns efeitos durante o decorrer das falas como palmas ou outros efeitos." - Participante P06 sobre os Efeitos Sonoros.

"... Então uma sugestão é a de continuar nessa linha, mas também seria bom ter temas mais descontraídos relacionados a tecnologia.” - Participante P02 sobre Temas Abordados.

"Poderiam falar sobre carreiras da programação trazendo as diversas áreas, tem 
Tabela 2. Respostas para as questões fechadas no questionário de avaliação

\begin{tabular}{|l|l|l|l|}
\hline Item Avaliado & Mediana & Mínimo & Máximo \\
\hline A qualidade do áudio & 4 & 3 & 5 \\
\hline A qualidade dos efeitos sonoros (música de fundo, vinheta e efeitos sonoros) & 5 & 4 & 5 \\
\hline O nível de interação dos apresentadores & 4 & 3 & 5 \\
\hline A relevância dos temas abordados para sua formação & 5 & 5 & 5 \\
\hline
\end{tabular}

muita gente que não sabe que existem algumas áreas dentro do curso" - Participante P10 sobre Temas Abordados.

Com relação ao objetivo geral estipulado com o projeto, os discentes foram questionados sobre sua opinião do podcast em relação a facilitar o acesso à informação e estimular a integração dos discentes. Nesse contexto, os discentes afirmaram que a iniciativa era relevante para o contexto de isolamento social, além de permitir atrair a comunidade ao estudo de temas de computação. Alguns trechos que exemplificam os pontos citados são apresentados a seguir.

"Gostei muito da ideia de um podcast de grupo PET sobre computação, a qualidade inicial já está muito boa, os assuntos são relevantes para o momento que os graduandos vivem, como dito anteriormente, a tendência do PodComp é somente crescer." Participante P06 sobre Avaliação Geral do Impacto do Podcast.

”... Gostaria de parabenizar a produção por botar em prática essa iniciativa que tem muito potencial de gerar contato do PET com a Computação UFMA. Mantenham-se firmes e confiantes.” - Participante P08 sobre Avaliação Geral do Impacto do Podcast.

\section{Conclusão}

Este artigo apresentou um relato de experiência da produção de conteúdo digital no contexto de podcasts. A comunidade acadêmica recepcionou o projeto de forma positiva e vários elogios foram encaminhados ao PETComp dentro e fora do curso de Ciência da Computação da UFMA.

Do ponto de vista dos discentes participantes do projeto, vale ressaltar que devido à falta de experiência na área comunicativa ou mesmo por falta de prática em apresentações, os discentes apresentadores relataram dificuldades no início das gravações. Além disso, eles indicaram que a etapa de teste que ocorreu nos primeiros meses foi fundamental para a evolução das interações desenvolvidas nos episódios e evolução nas técnicas de edição de áudio. Na visão dos discentes, a atividade permitiu que cada um pudesse se conhecer, pois com o passar do tempo, ao se ouvirem, puderam observar vícios de linguagens que em uma situação de fala cotidiana não seria muito fácil de serem percebidos. Nesse sentido, a experiência foi de grande ajuda para o desenvolvimento do grupo PETComp - UFMA, visto que o processo de elaboração de materiais proporcionou o aprendizado de novas tecnologias e ferramentas, o que poderá ser aproveitado em projetos e atividades futuras, como a realização de cursos, oficinas, eventos, entre outros. Adicionalmente, os processos criados e a organização definida pela equipe irão auxiliar na execução de futuros projetos onde seja necessário pesquisar metodologias e analisar propostas concorrentes, além de melhorar as habilidades de comunicação dos discentes.

O processo relatado e as lições aprendidas listadas nesse artigo narram as atividades conduzidas e mostram respostas obtidas por meio de um questionário com os 
discentes ouvintes, permitindo-se o acesso a um relato de experiência da criação dessa mídia, tendo em vista as dificuldades, aprendizados e dados que ajudam na organização desse material. Como trabalho futuro, pretende-se que o PodComp se torne uma atividade contínua durante todo o ano, salvo em períodos facultativos. Além disso, pretende-se alimentar as redes sociais semanalmente com conteúdos relacionados ao podcast, com o intuito de interagir e agregar valor aos ouvintes que poderão comentar, compartilhar e dar feedbacks através dessas redes. É desejo de todo o grupo PETComp UFMA que este trabalho possa diminuir o impacto que a COVID-19 vem trazendo para a comunidade, diminuindo o distanciamento entre os discentes e aumentando o engajamento através da troca de conhecimento investigado e repassado pelos discentes do grupo PETComp.

\section{Referências}

Barros, Á. G., de Souza, C. H. M., and Teixeira, R. (2020). Evolução das comunicações até a internet das coisas: A passagem para uma nova era da comunicação humana. Cadernos de Educação Básica, 5(3):260-280.

Bell, T., Cockburn, A., Wingkvist, A., and Green, R. (2007). Podcasts as a supplement in tertiary education: An experiment with two computer science courses. In Conference on Mobile Learning Technologies and Applications (MoLTA), page 70.

Chan-Olmsted, S. and Wang, R. (2020). Understanding podcast users: Consumption motives and behaviors. New Media \& Society, page 1461444820963776.

Farias, Priscila Fabiane \& da Silva, L. (2020). Ensino em tempos críticos: a criação de um podcast para promoção do diálogo crítico docente no contexto da pandemia da Covid-19. AtoZ: novas práticas em informação e conhecimento, 9(2):229-233.

Ferreira, C., Anjos, J., Normando, J., Castro, M., Odakura, V., Sacchi, R., and Barvinski, C. (2016). Uso de podcast para apoio a aprendizagem de algoritmos em curso de graduação em computação. In Anais dos Workshops do Congresso Brasileiro de Informática na Educação, volume 5, page 1208.

Geraldes, W. B. (2020). O ensino à distância através do uso de podcast para cursos técnicos e superiores no instituto federal de goiás. In Anais do CIET: EnPED: 2020(Congresso Internacional de Educação e Tecnologias - Encontro de Pesquisadores em Educação a Distância).

Gimenes, I. (2015). Os dilemas didáticos da Engenharia de Software. Revista da Sociedade Brasileira de ComputaçãoBrasil, Edição, 28.

Mota, P. A. and Coutinho, C. P. (2016). O podcast na educação musical: relato de uma experiência. Eduser-Revista de Educação, 1(1).

Phillips, B. (2017). Student-produced podcasts in language learning-exploring student perceptions of podcast activities. IAFOR Journal of Education.

Soares, E. d. F. A., de Lucena, L. B., de Andrade, G. L. C., dos Santos, W. S., de Oliveira, Á. G. G., Batista, L. M. d. S. D., Gomes, D. A., and de Lima, R. W. (2020). As atividades realizadas pelo pet no desenvolvimento do curso de ciência da computação. Brazilian Journal of Development, 6(7):43642-43656.

Vlachopoulos, D. (2020). Covid-19: threat or opportunity for online education? Higher Learning Research Communications, 10(1):2. 